\title{
Télescope
}

Revue d'analyse comparée en administration publique

\section{Analyse critique de la littérature scientifique portant sur l'innovation dans le secteur public : bilan et perspectives de recherche prometteuses}

\section{Nassera Touati et Jean-Louis Denis}

Volume 19, numéro 2, printemps 2013

L'innovation dans le secteur public : au-delà des discours

URI : https://id.erudit.org/iderudit/1023837ar

DOI : https://doi.org/10.7202/1023837ar

Aller au sommaire du numéro

Éditeur(s)

L’Observatoire de l'administration publique

ISSN

1929-3348 (numérique)

Découvrir la revue

Citer cet article

Touati, N. \& Denis, J.-L. (2013). Analyse critique de la littérature scientifique portant sur l'innovation dans le secteur public : bilan et perspectives de recherche prometteuses. Télescope, 19(2), 1-21.

https://doi.org/10.7202/1023837ar
Résumé de l'article

Cet article se propose de synthétiser l'état des connaissances sur l'innovation dans le secteur public en se centrant sur trois questions de recherche : la diffusion et l'adoption des innovations, leur génération et leur implantation. Les études ayant choisi une approche dite de variance se sont essentiellement intéressées à la question de l'adoption des innovations. Ce courant de recherche cherche à mettre en évidence les facteurs influençant l'adoption des innovations. Les enseignements tirés de ces travaux sont souvent contradictoires en raison des liens complexes entre variables. Les études ayant choisi une approche processuelle offrent une interprétation plus riche des processus d'innovation en étudiant les interactions dynamiques entre l'innovation et le contexte. Cela dit, selon les visions théoriques mobilisées, les travaux existants ont surtout insisté sur les enjeux de nature politique; peu de travaux ont porté sur les enjeux d'apprentissage, d'où l'intérêt de développer un agenda de recherche sur ces enjeux, en s'intéressant également aux enjeux d'ancrage des innovations. Enfin, il s'agirait aussi de s'attarder sur les innovations complexes qui impliquent plusieurs organisations interdépendantes. 


\title{
ANALYSE CRITIQUE DE LA LITTÉRATURE SCIENTIFIQUE PORTANT SUR L'INNOVATION DANS LE SECTEUR PUBLIC : BILAN ET PERSPECTIVES DE RECHERCHE PROMETTEUSES
}

\author{
Par Nassera Touati, Professeure, École nationale d'administration publique \\ nassera.touati@enap.ca \\ Et Jean-Louis Denis, Professeur, École nationale d'administration publique \\ jean-louis.denis@enap.ca
}

RÉSUMÉ Cet article se propose de synthétiser l'état des connaissances sur l'innovation dans le secteur public en se centrant sur trois questions de recherche : la diffusion et l'adoption des innovations, leur génération et leur implantation. Les études ayant choisi une approche dite de variance se sont essentiellement intéressées à la question de l'adoption des innovations. Ce courant de recherche cherche à mettre en évidence les facteurs influençant l'adoption des innovations. Les enseignements tirés de ces travaux sont souvent contradictoires en raison des liens complexes entre variables. Les études ayant choisi une approche processuelle offrent une interprétation plus riche des processus d'innovation en étudiant les interactions dynamiques entre l'innovation et le contexte. Cela dit, selon les visions théoriques mobilisées, les travaux existants ont surtout insisté sur les enjeux de nature politique; peu de travaux ont porté sur les enjeux d'apprentissage, d'où l'intérêt de développer un agenda de recherche sur ces enjeux, en s'intéressant également aux enjeux d'ancrage des innovations. Enfin, il s'agirait aussi de s'attarder sur les innovations complexes qui impliquent plusieurs organisations interdépendantes.

ABSTRACT This paper critically analysis the empirical studies concerning innovation in the public sector. It focuses on three research questions related to: the adoption and diffusion of innovations, their generation and implementation. Works, based on a variance approach, have essentially studied the adoption phenomenon: their aim is to identify factors influencing the adoption of innovation. These studies' results are often contradictory because of the complex relations between variables. Process based studies offer a better understanding of the innovation phenomenon: in particular, they show how the context and the actors' perceptions influence innovation processes. This being said, these studies rely, for the most part, on political theoretical frameworks and consequently neglect learning issues. We argue that the development of a research agenda tackling these issues would be useful. It would be also important to pay more attention to innovation sustainability issues. Finally, studying complex innovations, which involve multiple interdependent organisations, is crucial. 


\section{INTRODUCTION}

Dans un contexte de crise financière, d'explosion de la demande de services publics notamment en raison du vieillissement de la population, d'émergence de problèmes complexes (itinérance, réchauffement climatique, etc.) - caractérisé entre autres par une très forte interdépendance entre acteurs (Levin, Cashore, Bernstein et Auld, 2012) -, le thème de l'innovation semble s'imposer dans les discours sur la réforme du secteur public (Osborne et Brown, 2005; Bartos, 2003). En effet, l'innovation est censée améliorer la performance du gouvernement et des différents systèmes de services et apporter une solution adaptée à des problèmes complexes (Albury, 2011). S'il existe une certaine controverse quant à la définition de la notion d'innovation, en lien avec l'ampleur du changement qu'elle représente ou induit (Osborne et Brown, 2011), un certain consensus émerge, à savoir : "l'innovation serait un processus impliquant la génération, l'adoption et la diffusion de nouvelles idées qui produirait un changement "qualitatif" dans le contexte particulier d'une organisation ${ }^{1}$ " (Sorensen et Torfing, 2011, p. 8). Le changement en question concernerait concrètement les technologies, les structures, les pratiques organisationnelles et professionnelles, l'offre de services et, plus récemment, les arrangements interorganisationnels (Mandell et Steelman, 2010). Un changement qualitatif remet en question les schèmes cognitifs des acteurs, ainsi que leurs pratiques.

Par ailleurs, les analystes s'entendent pour dire que l'innovation dans le secteur public présente des particularités liées au contexte politique qui influence fortement les objectifs organisationnels et conditionne l'obtention des ressources (Halvorsen et autres, 2005). On souligne souvent les obstacles à l'innovation dans le secteur public liés à l'insuffisance de réelles mesures incitatives (Landeau, 1993), à la prolifération des règles et procédures (Barzelay, 1992), au fait que les gestionnaires ne sont pas impliqués dans l'élaboration des finalités de l'action (Moore, 1995), à l'aversion au risque des politiciens et des gestionnaires (Borins, 2001), à la contrainte imposée par une gestion de la performance non axée sur les résultats (Newman, 2001), etc. Au-delà de ce discours, les données empiriques montrent également que l'innovation est possible dans le secteur public (Earl, 2002, cité dans Windrum et Koch, 2008). Si l'on se réfère à l'évolution des services et politiques publics, il est clair que le secteur public a également fait l'objet d'innovations radicales (Sorensen et Torfing, 2011). Il suffit de penser à la création de services gouvernementaux en ligne, à l'élaboration de politiques de reconnaissance des organismes communautaires, aux pratiques novatrices de logement social (notamment avec un soutien dans la communauté), etc. Le problème ne réside donc pas dans l'absence d'innovation, mais dans le fait que celle-ci est épisodique et dépendante d'évènements " accidentels » (Eggers et Singh, 2009). Cette situation a suscité une prise de conscience quant à l'intérêt d'une nouvelle orientation en matière d'innovation dans le secteur public, dont l'objectif est de faire en sorte que l'innovation soit plus "systémique», se poursuive et s'étende à l'ensemble du secteur public. Comme le résume Albury (2011), l'un des problèmes majeurs en matière d'innovation a trait à la diffusion de ces dernières, ce qui a d'ailleurs encouragé certains États, tels le Canada, les États-Unis et la GrandeBretagne, à mettre en place des programmes de "récompense » de l'innovation (Bernier et autres, 2011; Borins, 2008; Hartley, 2008), pour inciter les organisations publiques à innover mais aussi pour permettre la diffusion de meilleures pratiques.

1 Toutes les traductions sont des auteurs. 
En dépit de son importance dans le contexte actuel, l'innovation dans le secteur public a relativement été peu étudiée : ce n'est qu'à la fin des années 1990, que l'on a vu croître l'intérêt de recherche pour ce thème (Borins, 2001).

Le présent article se propose de faire une analyse critique de la littérature. Soulignons que certaines recensions de la littérature scientifique portant sur ce thème existent déjà. Nous pouvons notamment citer le travail réalisé par Djellal et Galouj (2012) qui ont examiné la façon dont les principaux travaux réalisés durant deux décennies de recherche sur l'innovation dans les services prennent explicitement en compte l'innovation dans les services publics, en s'intéressant à la manière dont les perspectives théoriques existantes, qui renvoient à différentes conceptions de la relation entre les phénomènes d'innovation dans les services et d'innovations industrielles, traitent la problématique de l'innovation dans les services publics.

La présente analyse de la littérature scientifique vise à développer un regard critique sur l'innovation dans le secteur public, d'une part, en ne se limitant pas à l'innovation dans les services et d'autre part, en se penchant sur des questions de recherche différentes, qui seront explicitées un peu plus loin.

Par ailleurs, précisons qu'il ne s'agit pas d'une revue systématique, mais d'une revue « traditionnelle » qui ne cherche pas à évaluer la « robustesse » des preuves scientifiques :

«Ce type de revue de la littérature sélectionne le matériel sur lequel elle se base. Son principal objectif est de fournir une compréhension globale de l'état des connaissances et de mettre en évidence les éventuels besoins de développement de nouvelles recherches » (Cronin et autres, p. 38).

Notre recension se limite aux études empiriques ayant trait à l'innovation dans le secteur public, qui peuvent être classées en deux catégories, renvoyant à deux approches méthodologiques distinctes :

- la $1^{\text {re }}$ approche dite « approche de la variance » ambitionne d'identifier les déterminants de l'innovation;

- la 2e approche dite « approche processuelle » vise, comme son nom l'indique, à analyser les processus d'innovation.

Ces deux traditions de recherche ont caractérisé l'évolution du champ des études organisationnelles (Langley, 2007). Les études adoptant une approche de la variance (Mohr, 1982) sont utiles pour élaborer des modèles caractérisant les relations entre les variables associées à un phénomène. Cela étant, ces études ne permettent pas de comprendre les phénomènes complexes et d'orienter la prise de décision. À titre d'exemple, le constat d'une corrélation entre la taille des organisations et leur performance peut nous laisser croire que les petites organisations ont intérêt à fusionner pour améliorer leur performance. Une telle inférence ignore la complexité des processus de fusion (enjeux culturels, politiques) qui peut carrément compromettre la réalisation des économies d'échelle (Langley, 2007). Aussi, plusieurs chercheurs (Pettigrew, 1992; Langley et autres, 2003; Calnan et Ferlie, 2003, Poole et Van de Van, 2004) proposent une autre alternative pour l'étude des phénomènes qui prend acte de la nature dynamique de ces derniers. Il s'agit désormais de mieux comprendre comment les choses arrivent et pourquoi (Langley, 2007). L'analyse processuelle implique (Langley, 2007) : 1) de retracer l'évolution du phénomène et le déroulement des évènements dans le temps; 2) d'examiner comment les effets de l'action influencent les actions futures; 3) de s'attarder sur les pratiques des acteurs; 4) de prêter attention aux discours 
des acteurs; 5) de déstabiliser la stabilité, par exemple, en s'intéressant aux processus de transformation de l'identité des acteurs. Dans le champ de la recherche sur l'innovation, plusieurs visions théoriques ont été mobilisées pour comprendre les processus d'innovation. En guise de synthèse des travaux existants, Poole et Van de Van (2004) ont proposé une typologie qui met en avant quatre grandes perspectives théoriques, correspondant à des modes de production de changement différents : perspective dialectique (conflits); perspective téléologique (construction sociale); perspective évolutionniste (compétition); théories de cycle de vie (régulation). Comme on le montrera ultérieurement, du fait de la nature des organisations publiques, ce sont, essentiellement les perspectives dialectiques et du cycle de vie qui ont influencé les travaux de recherche.

Notre texte sera structuré comme suit :

- Dans la première partie, nous introduirons les concepts de base et les questions de recherche généralement traitées dans la littérature scientifique;

- Dans la deuxième partie, nous remettrons en question les liens entre l'innovation et l'amélioration de la performance; l'objectif étant de développer un regard plus critique sur l'innovation.

- Dans la troisième, nous résumerons et analyserons les principaux enseignements de l'approche dite de variance concernant l'adoption des innovations dans le secteur public;

- Dans la quatrième, nous nous intéresserons aux études dites processuelles pour une compréhension plus fine du phénomène de l'innovation;

- Dans la cinquième, nous dresserons un bref portrait des sujets de recherche émergents;

- Pour finir, dans la partie discussion, nous nous proposons d'amorcer une réflexion sur les voies de recherche prometteuses.

\section{L'INNOVATION : CONCEPTS DE BASE ET QUESTIONS DE RECHERCHE}

\section{Caractérisation des innovations}

La recherche sur l'innovation s'est beaucoup appuyée, du moins dans le cadre de l'approche dite de la variance, sur une caractérisation des innovations, l'objectif étant d'identifier les déterminants d'innovation par type d'innovation. Dans cette optique, plusieurs typologies ont été proposées; on peut citer celle de Hartley (2005) :

- Innovation de produit (ex. : nouvelle instrumentation dans un hôpital);

- Innovation sur le plan des services - nouvelles façons d'offrir les services (ex. : services en ligne);

- Innovation sur le plan des processus - nouvelle façon de concevoir les processus organisationnels (ex. : réorganisation administrative des processus back-office);

- Innovation sur le plan de la stratégie - nouveaux objectifs organisationnels (ex. : évolution vers une police communautaire);

- Innovation sur le plan du positionnement - nouveaux contextes ou utilisateurs;

- Innovation en matière de gouvernance - (ex. : nouvelles modalités de participation des citoyens);

- Innovation sur le plan de la rhétorique - nouveaux concepts (ex. : taxe carbone). 
En fait, ces typologies sont plus ou moins utiles car les innovations correspondent souvent à plusieurs types. Par contre, il est utile de retenir que les innovations immatérielles versus matérielles renvoient à des processus de nature différente. À titre d'exemple, les innovations au niveau des services sont influencées par les caractéristiques propres aux services (Drejer, 2004), entre autres le fait que les services sont coproduits avec les usagers. De la même manière, Moore et Hartley (2009) soutiennent que les innovations en matière de gouvernance (ex. : partenariat avec des acteurs privés pour l'exploitation des parcs) constituent un véritable défi pour la théorie sur l'innovation, et ce, encore une fois, en raison de la spécificité de ces innovations qui, d'une part, sont conçues et implantées à travers un réseau d'organisations et qui, d'autre part, impliquent aussi des changements dans les ressources utilisées par l'activité productrice, des processus de décision concernant le quoi produire et les normes utilisées pour l'évaluation de la performance du système de production. Ce type d'innovation requiert, entre autres, d'adapter les approches de leur évaluation: il ne s'agit plus uniquement d'apprécier l'effet de l'innovation sur l'efficacité ou l'efficience du système mais également de s'interroger sur l'équité du système, sachant que les innovations en matière de gouvernance ont des répercussions sur les responsabilités des différents acteurs dans la production des biens publics et sur les pouvoirs des uns et des autres dans les processus de décision.

Au-delà de la distinction qui existe entre les innovations matérielles et immatérielles, certains analystes suggèrent plutôt de distinguer les innovations « déjà faites » ailleurs, qui requièrent une adaptation au contexte local et donc aussi un changement des pratiques et des innovations à inventer (Barnett, 1953, cité dans Fernandez et Wise, 2010) car les enjeux, notamment en termes d'apprentissage, sont différents tout en étant présents dans les deux contextes. Mentionnons que les innovations dans le secteur public résultent souvent d'une "découverte » - du fait qu'elles existent déjà dans d'autres milieux - et non d'une invention. Ce fut le constat de plusieurs études qui ont exploité les données tirées des concours de récompense des organisations innovantes dans le secteur public (Altshuler et Zeggans, 1997).

\section{Les questions « classiques » de recherche sur l'innovation}

Étant donné la « prévalence » du modèle d'innovation par diffusion dans le secteur public, concomitant notamment au mouvement du nouveau management public, de nombreuses études se sont intéressées aux deux questions de recherche suivantes :

La diffusion des innovations : on examine ici le phénomène de diffusion d'une innovation au sein d'une population d'organisations, plus particulièrement le rythme d'adoption de l'innovation (vitesse) et le taux de diffusion (proportion d'individus d'un système social ayant adopté l'innovation). L'explication du phénomène de diffusion a fait appel à des «variables » telles que les attributs perçus des innovations (avantage relatif, compatibilité, complexité), les efforts promotionnels des agents de changement, la nature du système social (ex. : rôle des réseaux); etc.

- L'adoption des innovations: On s'intéresse ici aux différences entre organisations fortement et faiblement innovantes, sachant que la capacité d'innovation peut être appréciée de différentes manières (par exemple, en prenant ou non en considération la « rapidité » d'adoption). Mentionnons aussi que la définition de « l'adoption » peut varier d'un auteur à l'autre, certains incluant les aspects d'utilisation et de mise en 
œuvre dans la notion d'adoption. Pour « expliquer» le phénomène d'adoption, on a par exemple examiné le rôle joué par les caractéristiques de l'environnement organisationnel, des organisations elles-mêmes, de l'innovation, des individus.

Hormis ces deux questions, d'autres études ont plus particulièrement examiné les enjeux liés à la mise en œuvre et à l'ancrage des innovations. Néanmoins, comme le soulignent certains auteurs (Greenhalgh et autres, 2004; Piening, 2011), ces derniers enjeux ont été moins étudiés. À cela, s'ajoutent des questions de génération des innovations qui ont été dominées par la recherche sur l'entrepreneuriat « public».

Enfin, en privilégiant une vision non normative de l'innovation, basée sur l'hypothèse que l'innovation ne donne pas forcément lieu à une amélioration de la performance, certaines études ont analysé l'effet de l'innovation sur la performance organisationnelle.

\section{L'INNOVATION ET L'IMPACT SUR LA PERFORMANCE ORGANISATIONNELLE : QUELLES SONT LES ÉVIDENCES?}

Comme cela a été mentionné précédemment, plusieurs analystes invitent la communauté à se débarrasser d'une vision normative des innovations. Comme l'illustre la figure suivante, l'innovation ne va pas forcément de pair avec l'amélioration de la performance (Hartley, 2005, p. 31).

\begin{tabular}{|c|c|c|}
\hline \multirow{2}{*}{$\begin{array}{l}\text { Amélioration } \\
\text { performance }\end{array}$} & $\begin{array}{l}\text { Amélioration } \\
\text { sans } \\
\text { innovation } \\
\text { (amélioration } \\
\text { continue) }\end{array}$ & $\begin{array}{l}\text { Innovation et } \\
\text { amélioration }\end{array}$ \\
\hline & $\begin{array}{l}\text { Sans } \\
\text { amélioration et } \\
\text { sans innovation }\end{array}$ & $\begin{array}{l}\text { Innovation } \\
\text { sans } \\
\text { amélioration } \\
\text { (ex. : services } \\
\text { non désirés } \\
\text { par usagers) }\end{array}$ \\
\hline Faible & & Élevée \\
\hline
\end{tabular}

Les études associées au domaine de la recherche évaluative ont empiriquement observé les effets produits par des interventions dites novatrices. Ce courant de recherche illustre comment les problèmes de mise en œuvre des interventions (ex.: problème d'appropriation des nouvelles pratiques) peuvent ne pas donner lieu à une amélioration de la performance. Au-delà des problèmes de mise en œuvre, l'interaction entre le contexte et les interventions en question peut influencer la production des effets. À titre 
d'exemple, l'évaluation de PRISMA - une innovation développée au Québec visant à offrir aux aînés des services de santé et des services sociaux intégrés (coordonnés) notamment en vue de limiter la perte d'autonomie - montre des répercussions positives sur la satisfaction des usagers et l'utilisation des services de l'urgence, pour ne citer que ces deux effets. Malheureusement, l'effet sur les capacités fonctionnelles des usagers semble non concluant (Hebert et autres, 2010 cités dans Vedel et autres, 2011).

Au-delà des leçons que l'on peut tirer des recherches évaluatives portant sur les effets d'une intervention donnée (souvent sur des innovations de services), d'autres travaux se sont intéressés aux effets de l'adoption de différents types d'innovation à travers le temps. À titre d'illustration, Damanpour et autres (2009) se sont intéressés, dans le cadre d'une étude longitudinale basée sur des données s'étalant sur quatre années, aux effets produits par l'adoption de 3 types d'innovation (services, processus technologiques et processus administratifs) en se basant sur un échantillon de 428 organisations publiques de la GrandeBretagne. Cette étude, malgré ses nombreuses limites («contrôle » insuffisant de certaines variables environnementales et internes, etc.) semble montrer que c'est la combinaison de différents types d'innovation (technologiques et non technologiques) qui génère des gains de performance.

Pour approfondir la compréhension des liens entre l'adoption des innovations et les effets sur la performance, Walker et autres (2010) ont focalisé leur attention sur les effets produits (directement ou indirectement) par l'adoption d'innovations managériales : les auteurs postulent que les innovations managériales sont plus « réussies » lorsqu'il y a une cohérence entre les nouvelles pratiques qu'on introduit et les objectifs et indicateurs en vigueur dans l'organisation. Les auteurs, qui se sont basés sur une enquête portant sur 136 gouvernements locaux de la Grande-Bretagne, soutiennent que les innovations managériales n'ont pas de répercussion directe sur la performance organisationnelle et que cet effet est médiatisé par les pratiques de gestion de la performance.

En définitive, on peut affirmer que la recherche sur les effets des innovations en est à ses balbutiements : d'une part, peu de recherches portent sur les effets des innovations autres que les innovations de services; d'autre part, malgré les efforts déployés par certains auteurs, on ne saisit pas encore suffisamment la complexité du phénomène de production des effets : il s'agit de « contextualiser » davantage l'analyse des effets.

\section{LES PRINCIPAUX ENSEIGNEMENTS DE LA RECHERCHE SUR L'INNOVATION ADOPTANT UNE APPROCHE DITE DE VARIANCE : CONTRIBUTIONS ET LIMITES}

Plusieurs travaux se sont attachés à cibler les déterminants de l'adoption des innovations, surtout par les gouvernements locaux.

\section{Influence des variables internes}

Une série de travaux a notamment étudié l'influence des variables internes. À titre d'exemple, Damanpour et Schneider (2008) ont examiné l'influence des caractéristiques de l'innovation (complexité et coût) et des gestionnaires. Leur étude réalisée auprès de municipalités américaines a plus précisément analysé l'adoption (définie ici comme l'implanta- 
tion) de 25 pratiques organisationnelles «novatrices ». En contradiction avec l'hypothèse de départ, le coût de l'innovation ne semble pas décourager son adoption. De même, les liens entre la complexité de l'innovation et son adoption ne sont pas significatifs. Selon les auteurs, ces résultats suggèrent que les gestionnaires du secteur public accordent plus d'importance à l'effet présumé des innovations qu'à leur coût ou à leur complexité. Par ailleurs, la nature des innovations retenues dans l'étude (incrémentales et administratives) ferait en sorte que le coût et la complexité des innovations sont des critères moins importants. Cette étude semble aussi confirmer l'influence du niveau d'éducation des gestionnaires comme facteur facilitant l'adoption d'innovations. Par contre, dans le cadre de cette étude (et contrairement à d'autres), il ne semble pas y avoir de relation linéaire entre le nombre d'années d'expérience et l'adoption d'innovations (la relation est plutôt en forme de U).

Bhatti et autres (2011) ont aussi examiné le rôle des administrateurs professionnels dans le contexte municipal danois et suggèrent aussi que ces derniers favorisent l'adoption d'innovations (l'exemple étant le « Centre de services intégrés pour les citoyens »).

Au-delà du rôle de certains acteurs et de leur profil, une étude concernant l'innovation dans le secteur municipal australien semble montrer que le réseautage des acteurs joue un rôle déterminant (Considine et Lewis, 2007) : plus précisément, les acteurs bénéficiant d'une position centrale dans les réseaux d'information stratégique sont perçus comme des innovateurs et jouent un rôle actif dans les processus d'innovation.

Hansen (2011) a voulu pousser plus loin l'analyse en déterminant les facteurs internes qui influencent l'adoption de différents types d'innovations organisationnelles (privatisation, séparation du producteur et de l'acheteur, gestion par contrats, gestion par objectifs, etc.) associées au nouveau management public, et ce, dans différents contextes de gestion dans les municipalités danoises (gestion des écoles, gestion des services sociaux, etc.). Cette étude établit le constat que les déterminants varient en fonction du type d'innovation : ainsi, les gestionnaires dont les priorités sont de maintenir de « bonnes relations » avec leurs supérieurs et leurs pairs sont moins enclins à adopter les innovations associées au nouveau management public; excepté lorsqu'il s'agit d'innovations moins controversées (management par objectif, par exemple).

\section{Influence d'une combinaison de variables internes et externes}

Pour mieux saisir la complexité du phénomène d'adoption, des modèles plus sophistiqués intégrant aussi bien des variables internes qu'externes ont été développés. Par exemple, Fernandez et Wise (2010) se sont basés sur l'exemple de l'adoption d'une innovation en matière de dotation en personnel des postes de travail (visas pour le recrutement de travailleurs étrangers) par les districts scolaires du Texas pour montrer que différents facteurs favorisent l'adoption d'innovations de ce type, en l'occurrence : la marge de manœuvre en matière de ressources, la perception de faiblesses sur le plan de la performance par les gestionnaires, le rôle des leaders, l'ampleur de la demande pour des services spécialisés. En prenant l'exemple d'innovations technologiques, Tat-Kei Ho et Ya Ni (2004) ont aussi examiné l'influence de différentes variables internes (disponibilité de ressources, perception des acteurs concernant les services en ligne, support politique interne, etc.) et externes (pression exercée par le "monde » des affaires, progrès au niveau de ce type de développement dans le voisinage, etc.) sur l'adoption de solutions technologiques (création d'un site Web, ou développement de services en ligne plus sophistiqués). Les résultats de cette étude semblent montrer (en contradiction avec d'autres) que le soutien des élus a un impact 
important sur la décision d'exploiter les technologies de l'information. De même, les capacités (techniques, budget), la pression de l'environnement ne semblent pas associées au phénomène d'adoption, à la "surprise » des auteurs qui concluent que l'adoption de ce type d'innovation résulte davantage de l'influence du leadership politique, qui prend ses décisions en fonction de leurs perceptions des réactions du personnel.

Walker (2012) s'est attelé à réaliser une revue systématique des travaux qui ont étudié les déterminants (variables internes et externes) de l'adoption d'innovations de processus (associées à la manière dont les services sont produits) par des gouvernements locaux. Leur méta-analyse semble montrer que les variables internes importent davantage que les variables externes : la taille de l'organisation et l'intensité administrative, en particulier, semblent deux variables déterminantes. Par contre, les évidences ne sont pas fortes quant à l'influence de la marge de manœuvre en matière de ressources financières et de l'environnement institutionnel et technique.

Afin de mieux comprendre les phénomènes d'adoption associés à différents types d'innovation, Walker (2006) a testé, grâce à une enquête auprès de gouvernements locaux en Grande Bretagne, un modèle pour cinq types d'innovation (un correspondant à une innovation de produits; trois correspondant à des innovations de processus; un correspondant à une innovation inter-organisationnelle) et a conclu que différentes variables " expliquent» les phénomènes en question. À titre d'illustration, les innovations de produit sont davantage associées aux caractéristiques organisationnelles (leadership politique et petites organisations) et aux modèles de diffusion (pression du public; compétition) et moins aux variables d'environnement. À l'inverse, les innovations « organisationnelles » sont influencées par les variables d'environnement (changement des contextes social, politique et économique) et organisationnelles (leadership politique). Le leadership administratif ne s'avère pas une variable déterminante dans cette étude. Ces constats amènent l'auteur à conclure que l'adoption d'innovations est un phénomène complexe et contingent.

À travers son analyse des phénomènes d'adoption des innovations, Walker (2007) a aussi étudié comment, outre les variables environnementales et organisationnelles, l'adoption d'un type donné d'innovation influence l'adoption d'un autre type; l'hypothèse étant que l'innovation repose notamment sur la complémentarité entre types d'innovation : cette tentative a consisté à identifier les relations complexes entre variables, en mettant en évidence des configurations (des combinaisons). À titre d'exemple, il semble que les innovations de services soient associées à des organisations larges ayant des structures plus organiques (structures moins centralisées et formalisées) et situées dans des environnements plutôt simples, avec des niveaux élevés de besoins. Elles sont aussi soutenues par des innovations inter-organisationnelles et entravées par des innovations orientées vers l'amélioration de l'efficience du système de production. Les innovations organisationnelles (au niveau des processus administratifs, par exemple) se retrouvent plus dans les grandes organisations ayant des structures organiques et situées dans des environnements relativement complexes, avec des besoins de services élevés. Ce type d'innovation est davantage soutenu par les innovations de services et entravé par des innovations inter-organisationnelles. Walker mentionne que ces résultats ne sont pas entièrement conformes aux résultats d'autres études.

En définitive, si ces études contribuent à notre compréhension de la complexité du phénomène d'innovation, elles rencontrent plusieurs limites, notamment méthodologiques. L'une de ces limites découle du fait que les devis de recherche transversaux ne permettent 
pas d'établir des liens de causalité. Plusieurs auteurs soulignent aussi des problèmes de mesure, entre autres des mesures qui varient d'une étude à l'autre (par exemple, en ce qui a trait à la taille de l'organisation), ce qui fait qu'il est difficile d'accumuler des connaissances à partir des différentes recherches. Si des efforts ont été déployés pour développer des modèles de plus en plus sophistiqués, entre autres pour prendre en considération les relations pouvant exister entre divers types d'innovation, la non-prise en compte de l'évolution du phénomène dans le temps par des devis longitudinaux limite la validité de ces études.

Au-delà de ces limites méthodologiques, ces approches de variance qui sont amenées à associer les innovations étudiées, souvent multidimensionnelles, à un seul type sont discutables. De même, comme cela a été mentionné plus haut, ce courant de recherche ne s'intéresse pas à l'aspect processuel de l'innovation. Ici, l'innovation est davantage vue comme « une chose » qu'on introduit dans une organisation : par exemple, on ne prend pas en considération le fait que l'innovation et l'organisation qui l'adopte peuvent évoluer conjointement. Enfin, comme nous l'avons mentionné à quelques reprises, ces études aboutissent souvent à des résultats contradictoires et difficilement interprétables : la non-prise en compte du contexte de l'action ne permet pas de comprendre pleinement le phénomène à l'étude.

\section{LES PRINCIPAUX ENSEIGNEMENTS DE LA RECHERCHE SUR L'INNOVATION ADOPTANT UNE APPROCHE DITE PROCESSUELLE : CONTRIBUTIONS ET LIMITES}

Les études processuelles se sont efforcées de contribuer à une compréhension plus fine du phénomène d'innovation, en examinant les enjeux de diffusion, de production, et d'implantation des innovations.

\section{La diffusion des innovations}

Contrairement aux études inscrites dans le cadre de l'approche de la variance, les études processuelles se sont intéressées à l'évolution de l'innovation dans le cadre de sa diffusion. À titre d'exemple, Pope et autres (2006) ont examiné la diffusion d'une innovation organisationnelle visant à réduire les temps d'attente pour les chirurgies électives dans le contexte du National Health Service (Système de santé britannique). Cette étude documentait et analysait le sens conféré à cette innovation par les acteurs du système et son impact sur le développement de l'innovation. Elle illustre nomment les transformations du sens même de cette innovation et l'écart entre la vision gouvernementale et celle des acteurs locaux en regard du rôle du secteur privé, de l'ampleur du changement en question et de la " pertinence » de la création d'une capacité additionnelle. Ces écarts se traduisent par des variations sur le plan des formes concrètes d'implantation des centres de traitement au niveau local renvoyant à différentes stratégies d'acteurs (opportunistes, réalistes, idéalistes), par exemple en ce qui a trait à la révision des façons de faire.

De la même manière, des auteurs comme Fitzgerald et autres (2002) ont souhaité, en considérant le cas d'innovations dans le secteur de la santé, proposer une interprétation des processus de diffusion des innovations qui tient davantage compte de la nature non linéaire de ces processus. Leur étude se focalise en particulier sur trois aspects : le rôle des évidences scientifiques, la nature des décisions d'adoption et l'influence du contexte. De cette étude, plusieurs constats ressortent : 1) il n'y a guère d'association entre la robustesse des évi- 
dences et la vitesse de diffusion (contrairement à ce que laissent entendre certains analystes [Barnett et autres, 2011]). Les évidences font l'objet de jugement (en regard d'autres critères, telle la nature des incitations), et les décisions qui en découlent peuvent s'écarter du modèle « rationnel » de prise de décision; 2) les processus de diffusion sont interactifs et itératifs: les utilisateurs influencent l'innovation et sont eux-mêmes influencés par l'innovation. Au-delà de la réinvention et de la traduction des innovations, les résultats de cette étude suggèrent que les acteurs agissent en fonction de leurs propres intérêts et objectifs. À ce titre, cette recherche confirme que l'adoption d'innovation renvoie à un processus prolongé dans le temps, caractérisé par des négociations entre individus et groupes. La diffusion de l'innovation deviendrait alors possible lorsqu'une coalition de parties prenantes « influentes » génère un pouvoir «suffisant»; 3) enfin, encore une fois, l'importance du contexte (l'histoire et la culture organisationnelle, la qualité des relations entre acteurs, etc.) pour la compréhension du phénomène de diffusion est soulignée. Ce contexte est évolutif et est aussi le produit de l'action. D'autres travaux (voir, entre autres, Denis et autres, 2002) ont aussi montré que la diffusion des innovations dépend fortement de l'interaction entre la nature de l'innovation et le contexte (ex. : alignement de cette innovation avec les intérêts et les valeurs des acteurs).

Cela dit, pour interpréter ces processus de diffusion et d'adoption des innovations, deux cadres théoriques ont généralement été retenus : la théorie de l'acteur-réseau (Latour, 2005), renvoyant à une perspective dialectique et la théorie néo-institutionnelle (Dimaggio et Powell, 1983), essentiellement basée sur les perspectives de cycle de vie et dialectique (voir page 6).

Comme son nom l'indique, la théorie de l'acteur-réseau s'appuie sur deux notions centrales : soit le réseau et la négociation des controverses (Latour, 2005). Selon cette théorie, la diffusion d'une innovation serait fonction du degré de cohésion qu'elle suscite dans un réseau d'acteurs hétérogènes. Il s'agit dès lors de construire des relations d'alliances de façon à permettre une adaptation de l'innovation et du contexte dans lequel elle est introduite. Ceci passe par un travail de traduction de l'innovation. Ce cadre théorique a été par exemple utilisé par Tremblay (2007) pour étudier le déploiement du rôle d'infirmière pivot en oncologie (une innovation organisationnelle) dans le réseau de la santé du Québec. Cette étude montre en particulier que la diffusion de cette innovation exige que ses promoteurs adaptent les exigences de la fonction en fonction des capacités du milieu et des préoccupations des individus.

Les théories institutionnelles insistent, quant à elles, sur l'influence de l'environnement sur les organisations, à travers les institutions qui sont les règles, structures, mythes, qui donnent du sens et de la stabilité au comportement des acteurs. L'idée fondatrice de ces théories est que c'est davantage la recherche de la légitimité, plutôt que l'efficience, qui motive les comportements des acteurs. Ceci explique la convergence des structures organisationnelles (DiMaggio et Powell, 1983; Meyer et Rowan, 1977) dans un champ institutionnel, en l'occurrence une zone reconnue de la vie institutionnelle comprenant tous les acteurs pertinents (fournisseurs, producteurs, usagers, organismes de régulation, etc.). Les mécanismes permettant cet isomorphisme sont de trois types : mimétiques, normatifs et coercitifs. Verhoest, Verschuere et Bouckaert (2007) ont par exemple mobilisé cette théorie pour expliquer les comportements d'adoption des innovations dans le secteur public flamand : les études de cas réalisées par ces auteurs les amènent à soutenir que n'est pas seulement la compétition ou la pression pour la performance (les postulats de base du nouveau mana- 
gement public) qui incite les organisations publiques à innover. Des pressions, de nature plus politique, renvoyant à des enjeux de légitimité peuvent conduire les gestionnaires publiques à innover : lorsque les gestionnaires perçoivent un déclin sur le plan de l'appui politique ou de la société de façon plus générale, ils vont réagir dans certains cas en adoptant des innovations.

\section{La production des innovations}

Comme nous l'avons déjà mentionné, l'innovation dans le secteur public découle souvent de la diffusion des innovations issues du secteur privé; diffusion qui donne lieu à des adaptations locales (Sahlin-Anderson, 1996). Cela étant, les études empiriques révèlent aussi deux autres modes de production des innovations :

- L'innovation résulte d'initiatives prises par des entrepreneurs institutionnels qui vont agir comme des agents de changement. Les entrepreneurs institutionnels sont des acteurs disposant de ressources suffisantes pour générer de nouvelles institutions, notamment pour répondre à leurs intérêts (DiMaggio, 1988). L'intérêt pour l'entrepreneuriat en tant que moteur de l'innovation dans la recherche en administration publique, remonte à très longtemps (Windrum er Koch, 2008). Les travaux réalisés dans le contexte de l'administration publique, milieu fortement institutionnalisé caractérisé par une forte inertie, suggèrent que l'efficacité de l'entrepreneuriat passe par le déploiement d'un entrepreneuriat plus collectif ou plus systémique (Bernier et Hafsi, 2007) qui fait appel à des alliances entre acteurs. L'entrepreneuriat institutionnel est aussi favorisé par des contextes de crise : Fligstein (2001) a, par exemple, montré comment la crise au sein de l'Union européenne a permis à la Commission européenne d'agir comme entrepreneur et de développer le marché unique. Hormis l'influence du contexte sur la capacité d'agir, l'influence de la position de l'acteur (dans le champ institutionnel) sur ses comportements d'entrepreneur a fait l'objet de nombreux débats dans la littérature, la question étant : dans quelle mesure les intérêts et les opportunités présentes confèrent du pouvoir d'action à l'acteur? La recherche semble conclure que le changement émane souvent d'acteurs périphériques (Lockett et autres, 2012) car les acteurs sont : les moins avantagés par les arrangements institutionnels; les plus susceptibles de se départir des normes prévalentes; les plus en mesure d'être confrontés à des façons de faire alternatives. Cependant, les acteurs périphériques manquent généralement de pouvoir. À l'inverse les acteurs occupant une position centrale ont du pouvoir, mais sont généralement peu motivés à introduire le changement du fait des privilèges qu'ils retirent des arrangements institutionnels. À travers l'exemple des réformes dans le secteur de la santé, Lockett et autres (2012) montrent que ce sont les entrepreneurs qui occupent une position intermédiaire et qui combinent deux types de légitimité, en l'occurrence la légitimité structurelle et la légitimité morale, qui sont les mieux placés pour produire un changement radical. Pour renforcer leur légitimité, les entrepreneurs institutionnels sont amenés à développer des relations avec d'autres acteurs de façon à obtenir leur coopération, en s'appuyant sur des ressources matérielles (ex. : incitations) et symboliques (ex. : discours pour convaincre) (Hardy et Maguire, 2008). À ce titre, plusieurs auteurs considèrent que l'entrepreneuriat institutionnel renvoie à un processus hautement politique (Fligstein, 2001).

- L'innovation résulte d'activités de «bricolage » dans le cadre des tâches quotidiennes : ici, le rôle joué par les acteurs qui assument au quotidien la responsabilité du 
travail est essentiel (Andersen, 2008). Cette vision des processus de production des innovations met l'accent sur la nature incrémentale et plus ascendante du changement, lequel vise essentiellement à résoudre des problèmes en réemployant et en recombinant les actifs de l'organisation. Andersen (2008) montre, à travers quelques exemples empiriques, le rôle joué par la mobilisation de savoirs implicites et explicites et du capital social (voir les réseaux sociaux des acteurs) dans ces processus d'innovation.

\section{L'implantation et l'ancrage des innovations}

Les enjeux de mise en œuvre et d'ancrage des innovations ont, comme cela a déjà mentionné, été relativement peu étudiés. En fait, de nombreux travaux existants ont surtout tenté d'expliquer les difficultés d'implantation et d'ancrage, par exemple, des innovations managériales. C'est notamment le cas dans les bureaucraties professionnelles caractérisées par une forte autonomie des intervenants. En effet, il existe un écart entre les hypothèses implicites associées aux techniques managériales et les caractéristiques des bureaucraties professionnelles (Lozeau, Langley et Denis, 2002), écart qui fait en sorte que les techniques managériales sont souvent corrompues : on observe alors soit un faible couplage entre la technique et les dynamiques organisationnelles "réelles », soit une utilisation de la technique managériale reproduisant les structures de pouvoir. À titre d'illustration, les prémisses de la planification stratégique (influence forte du marché, autonomie des organisations, rôle important du sommet et d'une hiérarchie classique) ne se retrouvent pas dans les hôpitaux publics. De plus, les activités de planification sont détachées des vraies décisions stratégiques : le plan stratégique est davantage utilisé comme un outil promotionnel, et l'éclatement de la structure de pouvoir dans ces organisations fait en sorte que les plans stratégiques sont très vagues, résultant de négociations multiples entre acteurs.

Sans se référer au concept de corruption, d'autres auteurs ont mis en évidence les difficultés d'implantation des innovations, en faisant valoir qu'on assiste souvent à un phénomène de sédimentation (Cooper et autres, 1996). En analysant des cas de fusion d'hôpitaux universitaires aux États-Unis, Kitchener (2002) montre que cette fusion débouche sur certains comportements : certaines unités sont protégées de la fusion et la logique de fonctionnement en programme disparaît progressivement. Ces phénomènes de sédimentation donnent lieu à des structures instables qui, selon Kitchener, démontrent clairement les résultats décevants des innovations managériales. Ces phénomènes ont été décrits dans de nombreuses études qui ont notamment analysé la mise en œuvre de la réingénierie des processus et de l'évaluation de la performance dans les organisations publiques (McNulty et Ferlie, 2004; Mcgivern et Ferlie, 2007).

Ces difficultés de mise en œuvre et d'ancrage des techniques managériales sont généralement interprétées en s'appuyant sur les théories néo-institutionnelles, sachant que les organisations adoptent des innovations essentiellement pour se conformer aux pressions institutionnelles. Ces difficultés ont d'autant plus de probabilité de se manifester lorsque l'innovation en question ne fait pas de «sens » pour les acteurs, notamment parce qu'elle n'est pas accompagnée d'activités de sensegiving (Arnaboldi, Azzone et Palermo, 2010).

Au-delà de l'analyse de ces difficultés, d'autres auteurs se sont plutôt attachés à comprendre les dynamiques sous-tendant l'implantation des innovations. En s'appuyant sur des études de cas réalisées en milieu hospitalier, Piening (2011) a mis à jour les capacités 
dynamiques en œuvre dans ces processus, capacités qui sont nécessaires pour surmonter l'inertie organisationnelle. Par capacités dynamiques, on entend les capacités associées au changement et qui se traduisent par la mise en œuvre de routines d'apprentissage et de diffusion des connaissances.

Enfin, les enjeux de pérennisation des innovations suscitent aussi de plus en plus d'intérêt, notamment dans le domaine de la santé publique. Il faut savoir qu'il n'existe pas encore un consensus quant à la conception de la pérennisation. En effet, la revue de la littérature réalisée par Stirman et collaborateurs (2012) met en évidence la coexistence de deux grandes perspectives : 1) les modèles associés à la première perspective sont centrés sur l'étude de l'innovation en question. Ces modèles identifient les facteurs qui contribuent à la pérennisation, définie comme étant la fidélité, d'une innovation donnée; 2) les modèles associés à la seconde perspective sont de nature écologique et prennent en considération les liens entre l'innovation, son contexte d'implantation et l'environnement général. Cette seconde perspective met l'accent sur l'adaptation mutuelle entre le contexte et l'innovation, adaptation qui résulte notamment d'un processus d'amélioration continu (Chambers et autres, 2013). La pérennité d'une innovation serait dans ce cas la résultante de la cohérence entre l'innovation et son contexte. Les enjeux d'apprentissage organisationnel sont au cœur des processus de pérennisation des innovations, enjeux qui mériteraient certainement une étude plus approfondie (Chambers et autres, 2013). De façon générale, l'état actuel des connaissances ne permet guère de comprendre la complexité du phénomène de pérennisation des innovations (Stirman et autres, 2012) : les études existantes semblent montrer que plusieurs catégories de facteurs (contexte général [ex.: politiques], caractéristiques de l'innovation [ex. : adaptabilité], processus [ex. : processus d'évaluation], et capacités [ex. : financement]) sont importantes mais on ne saisit pas encore tout à fait comment l'interaction de ces facteurs favorise la pérennité de l'innovation (Stirman et autres, 2012).

En conclusion, nous pouvons affirmer que les études, dites de nature processuelle, offrent une interprétation riche des processus d'innovation, en remettant au centre de l'analyse le contexte d'action des acteurs. À la différence des approches dites de variance qui cherchent à « contrôler » l'effet du contexte, les approches processuelles prennent acte de la complexité du phénomène, notamment en étudiant les interactions dynamiques entre l'innovation et le contexte. À la différence aussi de l'approche dite de variance qui s'est concentrée sur les questions d'adoption des innovations, ce courant de recherche a abordé les questions de production et d'implantation des innovations. Cela dit, de par les visions théoriques mobilisées (théories de l'acteur réseau et théories néo-institutionnelles), les travaux existants ont surtout insisté sur les enjeux de nature politique; peu de travaux se sont intéressés aux enjeux d'apprentissage. Si certains ont souligné l'importance de la mobilisation des connaissances, ils n'ont pas, pour autant, abordé la question de production de nouvelles connaissances (Andersen, 2008).

De même, à notre connaissance, la majorité des travaux se situent dans des contextes intra-organisationnels. Or, la complexité des enjeux fait en sorte que l'innovation dans le secteur public requiert de plus en plus la collaboration de plusieurs organisations interdépendantes. Dans ces situations, le processus d'innovation est plus complexe: les actions entreprises par des entrepreneurs et les alliances créées entre acteurs ne suffisent pas pour produire l'innovation (Gherardi et Nicolini, 2005). Comme nous le verrons dans la section suivante, cette situation explique l'intérêt croissant des milieux de recherche à l'égard de l'innovation collaborative. 


\section{LES THÈMES DE RECHERCHE ÉMERGENTS : UN RAPIDE ÉTAT DES LIEUX}

Au-delà du bilan sur l'état des connaissances, la mise en évidence des sujets de recherche émergents nous semble également pertinente. Les tendances actuelles montrent un intérêt croissant pour :

\section{L'innovation collaborative}

L'implication des usagers dans les processus d'innovation

La gestion des risques dans le contexte de l'innovation

Les innovations en matière de gouvernance
Plusieurs auteurs suggèrent de s'intéresser davantage à la question des liens entre la collaboration et l'innovation (Bland et autres, 2010; Sorensen et Torfing, 2011; Sorensen et Torfing, 2012). Ces auteurs partent de l'hypothèse de l'existence d'un potentiel d'innovation collaborative, car la collaboration peut apporter une plus value à différents niveaux: génération de nouvelles idées (partage d'expériences), sélection d'idées (évaluation basée sur plusieurs perspectives); implantation (mobilisation des ressources) et dissémination des pratiques (création de réseaux sociaux et professionnels). Cela étant, ce potentiel peut ne pas se concrétiser en raison de problèmes de coordination (Swan et Scarbrough, 2005), de barrières cognitives (Ferlie et autres, 2005), de conflits d'intérêts, etc. Aussi, il serait nécessaire, selon ces auteurs, de mieux comprendre les conditions de production d'innovations dans des contextes distribués.

Le concept d'innovation ouverte renvoyant au renforcement du rôle des « utilisateurs » dans les processus d'innovation, développé initialement dans le monde industriel, commence aussi à pénétrer le secteur public (Hartmann et Hienerth, 2012; Kinder, 2012). Dans cette optique, certains travaux émergent autour des enjeux de conception de dispositifs (ex. : laboratoires) permettant une plus grande participation des différentes parties prenantes dans ces processus, incluant les usagers (Bason, 2012; Vibeke Cartensen et Bason, 2012). L'analyse du potentiel de ces dispositifs constitue une question centrale dans ce programme de recherche.

En constatant une tendance de minimisation des risques dans le secteur public, tendance pouvant nuire à l'innovation, Brown et Osborne (2012) invitent les gestionnaires publics à se départir d'une vision purement négative du risque et à se pencher sur la question de la gestion des risques, en privilégiant une vision constructive du risque basée notamment sur la négociation entre parties prenantes. Le développement de projets de recherche visant à mieux comprendre ces processus devrait aussi susciter l'intérêt de la communauté scientifique.

Étant donné la spécificité des innovations en matière de gouvernance (voir page 7) Moore et Hartley (2009) «militent» de leur côté pour une plus grande attention accordée à l'étude de ce type d'innovations. Sur la base de données empiriques, Borins (2008) confirme que les innovations en matière de gouvernance constituent la nouvelle génération d'innovations au sein du secteur public. 
Au-delà du renouvellement des thèmes de recherches, certains auteurs jugent qu'il serait également opportun de renouveler les processus de recherche, notamment en créant un plus grand dialogue entre les praticiens et les chercheurs (Wallis, 2011).

\section{- DISCUSSION ET CONCLUSION}

Cette recension de la littérature empirique sur l'innovation dans le secteur public met en évidence l'intérêt croissant pour le thème en question.

Ceci dit, comparativement à d'autres thèmes de recherche, nous pouvons affirmer que les enjeux relatifs à l'innovation ont été relativement peu étudiés. Ceci est d'autant plus vrai pour certains types d'innovations, tels que les innovations au niveau des services et de la gouvernance - des innovations qui sont à première vue moins tangibles et qui ne peuvent se limiter à des simples innovations technologiques. Cet état de fait peut être qualifié de paradoxal étant donné que les États sont de plus en plus confrontés au besoin de «faire plus avec moins ", situation qui requiert d'implanter des innovations dites "perturbatrices" (Eggers et autres, 2012). La notion d'innovation "perturbatrice » a initialement été introduite par Christensen (cité dans Eggers et autres, 2012) pour caractériser certains types d'innovation dans le secteur privé et décrit des «processus d'innovation qui résultent en la production de nouveaux produits ou services, qui prennent racine dans des applications simples développées à petite échelle, mais qui finissent par s'imposer dans le marché et supplanter les produits concurrents (notre traduction) ». Les innovations dites perturbatrices induisent des changements importants dans la logique de fonctionnement du système. Si l'on se réfère à l'exemple du système de la santé caractérisé par une forte inertie (Coiera, 2011), il est clair que le secteur public a de la difficulté à produire ce type d'innovations. Plusieurs phénomènes contribuent à ces difficultés. D'une part, des lacunes sur le plan de la diffusion des innovations, se traduisant soit par une non-diffusion ou une dilution des innovations due aux multiples négociations entre acteurs, empêchent de tels changements systémiques (Denis et autres). Les travaux actuels ne permettent pas vraiment de comprendre comment favoriser la diffusion des innovations, sans dilution. Il s'agit donc d'y remédier. D'autre part, la mise en œuvre et l'ancrage des innovations demeurent des enjeux majeurs dans le secteur public, sachant que l'adoption des innovations, du moins celles bouleversant les modes de fonctionnement usuels, est souvent superficielle. L'étude des conditions d'ancrage des innovations mérite certainement d'être aussi développée.

Par ailleurs, il faut savoir que les innovations perturbatrices exigent d'aller au-delà de l'innovation technologique, l'enjeu étant notamment d'associer à ces innovations technologiques de nouveaux modèles d'affaires. Ceci semble cohérent avec certains résultats de recherche qui montrent que la performance organisationnelle passe par la combinaison de différents types d'innovation (Damanpour et autres, 2009). Les données empiriques nous laissent penser que les organisations publiques ont justement parfois de la difficulté à aller au-delà des innovations technologiques : à titre d'illustration, l'implantation des technologies de l'information dans les organisations de santé ne suffit pas à donner lieu à des changements significatifs de pratiques professionnelles et organisationnelles. Par conséquent, il serait important de mieux saisir les processus par lesquels les avancées technologiques induisent des changements de pratiques.

Au-delà de l'analyse des enjeux susmentionnés, on ne peut faire l'économie de l'étude des processus d'émergence de l'innovation, sachant que cette dernière ne résulte pas uniquement des processus de diffusion. Il s'agirait notamment de prendre acte du fait que ces processus 
d'innovation impliquent désormais de nombreuses organisations interdépendantes, comme c'est le cas pour les enjeux liés au réchauffement climatique, à l'itinérance, etc. Ces processus sont hautement complexes : les alliances entre entrepreneurs ne constituent plus forcément des mécanismes suffisants pour la production du changement. Dans ces conditions, il est plutôt question d'organiser des écologies (Dougherty et Dunne, 2011) qui doivent notamment favoriser une orchestration de processus d'apprentissage interorganisationnel. Malheureusement, comme on a pu le constater, l'analyse empirique de ces processus d'apprentissage dans le contexte de l'innovation a, jusqu'à présent, très peu retenu l'attention des chercheurs.

En définitive, il semble que le développement, en collaboration avec des milieux de décision et de pratique, d'un véritable programme de recherche sur l'innovation dans le secteur public, portant sur les thèmes identifiés dans cet article, s'impose.

\section{- BIBLIOGRAPHIE}

Albury, D. (2011). «Creating the Conditions for Radical Public Service Innovation », Australian Journal of Public Administration, vol. 70, no 3, p. 227-235.

Altshuler, A. A. et M. D. Zegans (1997). «Innovation and public management: Notes from the state house and city hall », dans Altshuler, A. A. et R. D. Behn, (éd.), Innovations in American government. Challenges, Opportunities and dilemmas, Brookings.

Andersen, 0. J. (2008). «A Bottom-Up Perspective on Innovations: Mobilizing Knowledge and Social Capital Through Innovative Processes of Bricolage », Administration \& Society, vol. 40, no 1 , p. 54-78.

Arnaboldi, M., G. Azzone et T. Palermo (2010). « Managerial Innovations in Central Government: Not Wrong, but Hard to Explain », International Journal of Public Sector Management, vol. 23, no 1 , p. 78-93.

Balle Hansen, M. (2011). "Antecedents of Organizational Innovation: the Diffusion of New Public Management into Danish Local Government», Public Administration, vol. 89, n 2, p. 285-306.

Bartos, S. (2003). "Creating and Sustaining Innovation», Australian Journal of Public Administration, vol. 62, no 1, p. 9-14.

Barnett, J. et autres (2011). « Understanding Innovators' Experiences of Barriers and Facilitators in Implementation and Diffusion of Healthcare Service Innovations: a Qualitative Study», BMC Health Services Research, vol. 11, no 1, p. 1-12.

Barzelay, M. et B. J. Armajani (1992). Breaking through bureaucracy: a new vision for managing in government, Berkeley, University of California Press.

Bason, C. (2012). "Design as catalyst of new organisational readings", texte présenté à l'International Research Society for Public Management XVI Conference, Rome, Italie, Avril.

Bernier, L., et T. Hafsi (2007). "The changing nature of public entrepreneurship », Public Administration Review, vol. 67, $\mathrm{n}^{\circ}$ 3, p. 488-503.

Bernier, L., T. Hafsi et C. Deschamps (2011). Innovation in the Public Sector: A Look at The Innovation Award of the Institute of Public Administration of Canada Applications and Winners, Québec, Centre de recherche sur la gouvernance, ENAP. 
Bhatti, Y., A. L. Olsen et L. Holm Pedersen (2011). "Administrative Professionals and the Diffusion of Innovations: the Case of Citizen Service Centres », Public Administration, vol. 89, $\mathrm{n}^{\circ} 2$, p. 577-594.

Bland T. et autres (2010). "Enhancing Public Sector Innovation: Examining the NetworkInnovation Relationship ", The Innovation Journal: The Public Sector Innovation Journal, vol. 15, no 3, p. 1-17.

Borins, S. (2001). « Encouraging Innovation in the Public Sector», Journal of intellectual capital, vol. 2, n 3 , p. 310-319.

Borins, S. (2008). Innovations in Government: Research, Recognition, and Replication, Washington, D.C., Brookings Institution Press.

Calnan, M. et E. Ferlie (2003). «Analysing Process in Healthcare: The Methodological and Theoretical Challenges », Policy \& Politics, vol. 31, no 2, p. 185-193.

Chambers, D. A., R. E. Glasgow et K. C. Stange (2013). « The dynamic Sustainability Framework: Addressing the Paradox of Sustainment Amid Ongoing Change », Implementation Science, vol. 8, no 1 .

Cooper, D. J. et autres (1996). "Sedimentation and Transformation in Organizational Change: The Case of Canadian Law Firms », Organization Studies, vol. 17, no 4, p. 623-647.

Brown, L., et S. Osborne (2012), «Risk and Innovation Towards a framework for risk governance in public services », Public Management Review, vol. 15, n 2, p. 186-208.

Considine, M. et J. M. Lewis (2007). «Innovation and Innovators Inside Government: From Institutions to Networks », Governance: An International Journal of Policy, Administration and Institutions, vol. 20, no 4, p. 581-607.

Denis, J-L. et autres (2002). «Explaining diffusion patterns for complex health care innovations », Health Care Management Review, vol. 27, n 3, p. 60-73.

DiMaggio, P. J. (1988). «Interest and agency in institutional theory », dans Zucker, Institutional patterns and culture, Cambridge MA, Ballinger Publishing Company.

DiMaggio, P. J. et W. W. Powell (1983). « The iron cage revisited: institutional isomorphism and collective rationality in organizational fields », American Sociological review, vol. 48, $\mathrm{n}^{\circ} 2$, p. 147-160.

Dougherty, D., et D. Dunne (2011). « Organizing ecologies of complex innovation », Organization Science, vol. 22, no 5, p. 1214-1223.

Drejer, I. (2004). «Identifying innovation in surveys of services: a Schumpeterian perspective », Research Policy, vol. 33, no 3, p. 551-562.

Eggers, B., et S. Singh (2009). The public innovators playbook. Washington, DC:Harvard Kennedy School of government.

Eggers, W. et autres (2012). "Disruptive innovation: a new model for public sector services », Strategy \& Leadership, vol. 40, no 3, p. 17-24.

Fernandez, S. et L. R. Wise (2010). "An Exploration of why Public Organizations "Ingest" Innovations », Public Administration, vol. 88, nº 4, p. 979-998.

Fitzgerald, L. et autres (2002). «Interlocking Interactions, the Diffusion of Innovations in Health Care », Human Relations, vol. 55, no 12, p. 1429-1449.

Fligsten, N. (2001). "Institutional entrepreneurs and cultural frames: the case of the European Union's single market program », European societies, vol. 3, n³ 3, p. 261-287. 
Ferlie, E. et autres (2005). " The nonspread of innovations: the mediating role of professionals », Academy of Management Journal, vol 48, n 1, p. 117-134.

Gherardi, S., et D. Nicolini (2005). "Actor-Networks: Ecology and Entrepreneurs », dans B. Czarniawska et T. Hernes (éd.), Actor-Network Theory and Organizing. Copenhague, Liber, p. 285-306.

Greenhalgh, T. et autres (2004). «Diffusion of Innovations in Service Organizations: Systematic Review and Recommendations », The Milbank Quarterly, vol. 82, n 4, p. 581-629.

Halvorsen, T. et autres (2005). «On the Differences Between Public and Private Sector Innovation », Communication présentée à NIFU STEP, Oslo, Publin report no D9.

Hardy, C., et S. Maguire (2008). «Institutional entrepreneurship ». dans Greenwood et autres. The Sage Handbook of organizational institutionalism, Sage, p. 198-217.

Hargadon, A. et R. J. Sutton (2003). "Créer un laboratoire d'innovation», dans A. Dutheil et autres, Les meilleurs articles de Harvard Business Review sur l'innovation, Paris, Éditions d'Organisation, p. 65-91.

Hartley, J. (2005). «Innovation in Governance and Public Services: Past and Present », Public Money \& Management, vol. 25, nº 1, p. 27-34.

Hartley, J. (2008). « Does Innovation Lead to Improvement in Public Services? Lessons from the Beacon Scheme in the United Kingdom », dans S. Borins, Innovations in Government: Research, Recognition, and Replication, Washington, D.C., Brookings Institution Press, p. 159 à 187.

Hartmann, R. K. et C. Hienerth (2012). «Open innovation in regulation of public services: exploring varieties in formes of openness to regulates and self regulators ». Texte présenté à l'International Research Society for Public Management XVI Conference, Rome, Italie, Avril.

Kinder, T. (2012). « Learning, Innovating and Performance in Post-New Public Management of Locally Delivered Public Services », Public Management Review, vol. 14, no 3, p. 403-428.

Kitchener, M. (2002). « Mobilizing the Logic of Managerialism in Professional Fields: The Case of Academic Health Centre Mergers », Organization Studies, vol. 23, nº 3, p. 391-420.

Koss Hartmann, R. et C. Hienerth (2012). «Open Innovation in Regulation of Public Services: Exploring Varieties in Forms of Openness to Regulatees and Self-Regulators », Communication présentée à l'International Research Society for Public Management XVI Conference, Rome, Italie, Avril.

Landeau, J. (1993). «Organizational change and barriers to innovation: A case study in The Italian Public Sector », Human Relations, vol. 46, n 12, p. 1411-1429.

Lockett, A. et autres (2012). "The Role of Institutional Entrepreneurs in Reforming Healthcare », Social Science \& Medecine, vol. 74, n³ , p. 356-363.

Lozeau, D, A. Langley, et J.-L. Denis, (2002). «The Corruption of Managerial Techniques by Organizations », Human Relations, vol. 55, n 5, p. 537-564.

Mandell, M. et T. Steelman (2003). «Understanding What can be Accomplished Through Interorganizational Innovations: the Importance of Typologies, Context and Management Strategies », Public Management Review, vol. 5, n² 2, p. 197-224.

Manoharan, A. (2012). "A Study of the Determinants of County E-Government in the United States », American Review of Public Administration, vol. 43, n 2, p. 159-178. 
Meyer. J. W. et B. Rowan (1977). «Institutionalized Organizations: Formal Structures as Myth and Ceremony », American Journal of Sociology, vol. 83, n 2, p. 340-363.

McGivern, G. et E., Ferlie (2007). « Playing tick-box games: Interrelating defences in professional appraisal », Human Relations, vol. 60, nº 9, p. 1361-1385.

McNulty, T. et E. Ferlie (2004). « Process Transformation: Limitations to Radical Organizational Change within Public Service Organizations », Organization Studies, vol. 25, no 8, p. 13891409.

Moore, M. H. (1995). Creating public value: strategic management in government, Harvard University Press.

Moore, M. et J. Hartley (2009). «Innovations in Governance», dans S. P. Osborne (ed.), The New Public Governance? Emerging Perspectives on the Theory and Practice of Public Governance, New York, Routledge, p. 52-71.

Newman, J. et autres (2001). "Transforming local government: innovation and modernization », Public money \& management, vol. 21, $\mathrm{n}^{\circ}$ 2, p. 61-68.

Osborne, S. P. et L. Brown (2011). «Innovation, Public Policy and Public Services Delivery in the UK: The World that would be King? », Public Administration, vol. 89, n 4, p. 1335-1350.

Osborne, S. P. et K. Brown (2005). Managing Change and Innovation in Public Service Organizations, New York, Routledge Taylor \& Francis Group.

Pettigrew, A. M. (1992). "The Character and Significance of Strategy Process Research», Strategic Management Journal, vol. 13 (winter special issue), p. 5-16.

Piening E. P. (2011). "Insights into the Process Dynamics of Innovation Implementation», Public Management Review, vol. 13, no 1, p. 127-157.

Poole, M. S., et A. Van de Ven (2004). Handbook of organizational change and innovation, Oxford University Press.

Pope, C. et autres (2006). « Lost in Translation: a Multi-Level Case Study of the Metamorphosis of Meanings and Action in Public Sector Organizational Innovation », Public Administration, vol. 84, no 1 , p. 59-79.

Sahlin-Andersson, K. (1996). «Imitating by editing success: the construction of organizational fields », dans B. Czarniawska et G. Sevon, Translating organizational change, Berlin New York, Walter de Gruyter, p. 69-92.

Sorensen, E. et J. Torfing (2012). "Collaborative Innovation in the Public Sector», The Innovation Journal: The Public Sector Innovation Journal, vol. 17, no 1, p. 1-14.

Sorensen, E. et J. Torfing (2011). "Enhancing Collaborative Innovation in the Public Sector», Administration \& Society, vol. 43, n 8 , p. 842-868.

Stirman, W. et autres (2012). "The sustainability of new programs and innovations: a review of the empirical literature and recommendations for future research », Implementation science, vol. $7, \mathrm{n}^{\circ} 17$.

Swan, J.A. et H. Scarbrough (2005). «The politics of networked innovation », Human Relations, vol. 58, n 7, p. 913-943.

Tat-Kei Ho, A. et A. Ya Ni (2004). "Explaining the Adoption of E-Government Features: A case Study of lowa County Treasures' Offices ", American Review of Public Administration, vol. 34, $n^{\circ} 2$, p. 164-180. 
Tremblay, D. (2007). La traduction d'une innovation organisationnelle dans les pratiques professionnelles de réseau: l'infirmière pivot en oncologie. Thèse de doctorat, Université de Montréal.

Vedel, I. et autres (2011). « Ten years of integrated care: backwards and forwards. The case of the province of Quebec, Canada », International journal of Integrated Care, vol. 11, n 7, p. 1-11.

Vibeke Carstensen, H. et C. Bason (2012). "Powering Collaborative Policy Innovation: Can Innovation Labs Help? », The Innovation Journal: The Public Sector Innovation Journal, vol. 17, no 1, p. 1-26.

Verhoest, K, B. Verschuere et G. Bouckaert (2007). «Pressure, Legitimacy, and Innovation Behavior by Public Organizations », Governance: An International Journal of Policy, Administration and Institutions, vol. 20, no 3, p. 469-497.

Walker R. M. (2006). "Innovation Type and Diffusion: an Empirical Analysis of Local Government », Public Administration, vol. 84, nº 2, p. 311-335.

Walker R. M. (2007). «An Empirical Evaluation of Innovation Types and Organizational and Environmental Characteristics: Towards a Configuration Framework», Journal of Public Administration Research and Theory, vol. 18, no 4, p. 591-615.

Walker, R. M., F. Damanpour et C. Devece, (2010). « Management innovation and organizational performance: the mediating effect of performance management», Journal of Public Administration Research and Theory, vol. 21, no 4, p. 367-386.

Walker, R. M. (2012). «Internal and External Antecedents of Process Innovation: A Review and Extension", Communication présentée à l'International Research Society for Public Management XVI Conference, Rome, Italie, Avril.

Windrum, P. et P. Koch (2008). Innovation in Public Sector Services: Entrepreneurship, Creativity and Management, Cheltenham, Royaume-Uni, Edward Elgar Publishing Limited. 